\title{
Treating Early-Stage DLBCL on the FLYER: What Lesson for Radiation Therapy?
}

\author{
Kelsey Sokol ", Amanda McBride, Adam Finn Binder and Pierluigi Porcu \\ Department of Medical Oncology, Division of Hematologic Malignancies and Hematopoietic Stem Cell Transplantation, \\ Sidney Kimmel Cancer Center at Jefferson Health, Thomas Jefferson University, Philadelphia, PA, United States
}

Keywords: DLBCL, favorable, early-stage, lymphoma, CHOP (R-CHOP), radiation

\section{INTRODUCTION}

The combination of cyclophosphamide, doxorubicin, vincristine, and prednisone (CHOP) and the anti-CD20 monoclonal antibody rituximab has been standard of care for diffuse large B-cell lymphoma (DLBCL) since 2006, based on results from three randomized Phase III clinical trials: GELA/LNH 98-5, Intergroup/E4494, and MInT (1-3). These trials showed that the addition of rituximab to 6-8 cycles of anthracycline-based chemotherapy increased overall survival (OS) and progression free (PFS) or event free survival (EFS) in both older patients $(>60)$ with advanced-stage DLBCL (GELA/LNH-98-5 and E4494), and younger patients $(\leq 60)$ with favorable-risk disease, defined as stage II-IV with 0-1 International Prognostic Index (IPI) risk factors or bulky stage I disease (MInT).

The MInT trial showed that 6 cycles of R-CHOP every 21 days (R-CHOP-21) are sufficient in younger DLBCL patients with favorable features and that patients with early-stage DLBCL (Ann Arbor I and II) and no tumor bulk (variably defined) had excellent outcomes without radiation therapy (RT), making R-CHOP x 6 an alternative to R-CHOP x $3+\mathrm{RT}$ (3). A subset of patients $(\mathrm{N}=101)$ with age-adjusted IPI 0 and disease bulk $<7.5 \mathrm{~cm}$ had very favorable outcomes (PFS and OS at 6 years $89.6 \%$ and $94.9 \%$, respectively). Did this population actually need 6 cycles of R-CHOP? Or could fewer cycles be sufficient (without RT)? While the FLYER trial was not designed to directly answer the RT question, it is relevant for RT decisions in clinical practice.

The FLYER trial was an international, randomized Phase III study conducted between December 2005 and October 2016 in the very favorable subset identified in the MInT trial, i.e. "young" patients (age 18-60) with stage I/II (based on CT or PET/CT) untreated CD20-positive aggressive B-cell lymphoma, without bulky disease, and with no age-adjusted IPI risk factors (4). The study enrolled 592 patients, of which $85 \%$ had DLBCL. Patients were randomized to receive R-CHOP every 21 days for 4 cycles, followed by an additional two doses of rituximab $(n=297)$, or six cycles of R-CHOP every 21 days $(n=295)$. Radiation was planned only in patients with testicular involvement, as prophylaxis to the contralateral testis.

The study asked whether a reduction in chemotherapy to 4 cycles would be non-inferior to standard treatment with 6 cycles, with a non-inferiority margin of $-5.5 \%$. After median follow-up of 66 months, the study met its primary endpoint, with an absolute difference in 3-yr PFS of 3\% (96\% with 4 cycles R-CHOP plus two doses of rituximab vs $94 \%$ with 6 cycles R-CHOP) and no difference in 3 -yr EFS or OS (89\% vs $89 \%$ and $99 \%$ vs $98 \%$, respectively). Forty patients progressed or relapsed. Rates of relapse for patients with complete response $(\mathrm{CR})$ or unconfirmed complete response $(\mathrm{CRu})$, 
based on the 1999 consensus criteria (5), were similar, with no CNS relapses. About two thirds of relapses occurred after 2 years. Fewer adverse events occurred in the reduced chemotherapy group. The rate of secondary neoplasm was similar. Does the FLYER study give us justification to use 4 cycles of R-CHOP, as opposed to 6 (as in MInT) for these patients? An important question that the FLYER study did not directly address is: can we treat some patients with early-stage DLBCL with chemotherapy only, omitting radiation?

\section{POINT}

The FLYER trial presents an improved treatment approach for younger patients with early-stage favorable risk DLBCL, decreasing toxicity through radiation omission and abbreviation of chemotherapy.

Prior to this trial, the addition of involved field RT to chemotherapy, or combined-modality therapy (CMT), was employed to allow for abbreviated chemotherapy in early-stage DLBCL. The phase III SWOG 8736 trial initially examined CMT in early-stage disease (6). Patients were randomized to 3 cycles CHOP followed by RT (40-55 Gy to all visible sites of disease) versus 8 cycles CHOP alone. Significant improvement in 4-year PFS (77\% vs $64 \%)$ and OS ( $82 \%$ vs $72 \%$ ) in favor of CMT was shown. This led to the widespread adoption of CMT for patients with early-stage DLBCL. The subsequent SWOG 0014 trial, a single arm phase II trial, confirmed excellent outcomes with CMT for early-stage DLBCL in the rituximab era (7).

Others evaluated radiation omission in early-stage DLBCL. The GELA LNH-93-4 trial examined abbreviated chemotherapy without RT in the pre-rituximab era. Patients with early-stage DLBCL and no adverse IPI risk factors were randomized to 4 cycles CHOP followed by RT versus 4 cycles CHOP alone (1). There was no difference in 5-year EFS or OS between the two groups. Notably, patients were $>60$ years old, a population excluded from FLYER. In the rituximab era, LYSA/GOELAM 02-03 randomized patients age 18-75 years with stage I/II nonbulky $(<7 \mathrm{~cm})$ disease to receive $\mathrm{RT}$ versus no RT if interim PET/ CT (iPET) following 4 cycles R-CHOP showed CR (88\% of patients) (8). Patients received a total of $4(n=187)$ or 6 cycles $(n=148)$ based on the presence of IPI risk factors. Notably, RCHOP was given every 14 days. For patients with CR on iPET, 5year EFS and OS were similar between patients who received CMT versus chemoimmunotherapy alone. Collectively, these trials support RT omission and chemotherapy abbreviation early-stage DLBCL.

Recently, the $\mathrm{S} 1001$ trial evaluated a response-adaptive strategy in patients with early-stage DLBCL (9). In this phase II study, 132 eligible patients with early-stage, nonbulky $(<10 \mathrm{~cm})$ disease received 3 cycles R-CHOP followed by iPET. If iPET negative, patients received 1 additional cycle $\mathrm{R}-\mathrm{CHOP}$ for 4 cycles total. If iPET positive, they received RT followed by ibritumomab tiuxetan, an anti-CD20 radioimmunoconjugate. In 128 iPET-evaluable patients, the 5-year PFS and OS were excellent in both iPET-pos $(\mathrm{N}=14)$ and iPET-neg $(\mathrm{N}=114)$ patients at $86 \%$ vs. $89 \%$ and $85 \%$ vs. $91 \%$, respectively.
Molecular and biologic risk factors were not evaluated in the FLYER trial. However, while cell of origin (COO) and double expressor (DE)/double hit (DH) status are important prognostic factors in advanced-stage DLBCL, the prognostic implication in early-stage is unclear. Three retrospective studies provide some data to address this question. One international study evaluated 192 patients with early-stage DLBCL treated with R-CHOP-like regimens with or without RT (10). Of these patients, $60 \%$ were classified as GCB and $40 \%$ as non-GCB. The total cohort had excellent outcomes with 4-year PFS 85\% and 4-year OS 88\%, and $\mathrm{COO}$ status did not influence outcomes. This study also evaluated outcomes related to $\mathrm{DE} / \mathrm{DH}$ status. Although limited numbers, DE (32 patients) and DH (6 patients) status was not associated with inferior PFS or OS. An additional retrospective analysis evaluated the impact of $\mathrm{COO}$ on outcomes in 87 patients with early-stage DLBCL treated with R-CHOP 3-4 cycles plus RT (11). Most patients (75\%) had GCB phenotype, and IPI was similar between GCB and non-GCB groups. Again, no significant difference in PFS or OS between the two phenotypes was found. More recently, a US multicenter retrospective study evaluated outcomes in 104 patients with MYC-rearranged earlystage DLBCL (including 40 patients with $\mathrm{DH}$ ) treated with standard R-CHOP vs more intensive immunochemotherapy (IIC = R-DA-EPOCH, R-HyperCVAD/MA, or R-CODOX$\mathrm{M} / \mathrm{IVAC})+/-\mathrm{RT}$. The 2 -year PFS and OS were $78 \%$ and $86 \%$ for the entire cohort, and $74 \%$ and $81 \%$ for the $\mathrm{DH}$ patients, respectively. PFS and OS were similar across treatment groups in the entire cohort and in patients with $\mathrm{DH}$, noting that the addition of consolidative RT yielded a higher CR rate. Furthermore, the ORR in $\mathrm{DH}$ patients was comparable between regimens (12). These studies suggest that $\mathrm{COO}$ and $\mathrm{DE} / \mathrm{DH}$ status may not have significant prognostic implications in early-stage DLBCL. Therefore, the lack of molecular and COO data in FLYER should not affect the final conclusions of the study.

\section{COUNTERPOINT}

For patients with early-stage, low-risk DLBCL, 3 cycles R-CHOP followed by involved field RT is an established and widely used therapeutic option to reduce chemotherapy exposure and toxicity. Avoidance of radiation and its associated toxicities is an arguable benefit of the FLYER trial; however, it is important to accurately assess this risk in modern times. While the location of the nodal disease dictates potential toxicities, current techniques allow for refined fields of radiation and reduction in dose, minimizing short-term toxicities such as mucositis and cytopenias. Data regarding long-term toxicities, including secondary malignancy, infertility, and cardiac dysfunction, is often extrapolated from Hodgkin lymphoma (HL) given the higher utilization rates of RT. Patients with HL are typically younger at diagnosis with more life-years for development of a secondary malignancy, and often receive mediastinal radiation. This is in contrast to the older population of DLBCL (median age 66 years) with smaller radiation fields often excluding major organ systems. That being said, there are special circumstances 
that must be considered on an individual basis in which radiation should be avoided, such as young female patients with inguinal nodal disease to preserve fertility as well as breast and mediastinal involvement of lymphoma.

The effect of COO and high-risk molecular features in earlystage disease is inconclusive and limited to retrospective studies. Several studies as described above found no different between subtypes. An additional retrospective review of 151 patients with early-stage disease evaluated $\mathrm{COO}$ in patients who received RCHOP with or without RT (13). COO was determined in 87 patients, with 62 GCB and 25 non-GCB. Excellent responses were observed in both therapeutic groups as expected. However, analysis of COO impact showed that non-GCB was associated with inferior 5 -year PFS and OS (73.9\% and 59.7\%) compared to GCB (95.9\% and $90.6 \%)$. The trend of inferior outcome was more pronounced in non-GCB receiving 4 cycles or less of R-CHOP regardless of RT. The authors of the FLYER trial plan to report biologic risk factors in their population of patients, a necessary analysis to support the safety of abbreviated therapy across all subtypes.

Long-term follow up from both the SWOG 8736 and SWOG 0014 trials shows a continued pattern of late relapses in early-stage DLBCL, suggesting that CMT and rituximab may not mitigate risk of long term relapse in this population $(7,14)$. This pattern was again observed in the FLYER study. While relapse rates after CR or unconfirmed CR were low at 4-5\%, the majority of relapses were observed after 2 years, with the cumulative incidence of relapse demonstrating a linear trend during years 3 to 6 of follow-up. So, while the approach of abbreviated chemotherapy was demonstrated to be non-inferior to a longer course of chemotherapy, the issue of late relapse in this early-stage, favorable-risk population remains.

The extended recruitment period (11 years) should be noted and was recognized by the authors. This prolonged recruitment time is likely reflective of the low incidence of early-stage, lowrisk DLBCL and of DLBCL in younger patients in general. While DLBCL is the most common type of non-Hodgkin lymphoma, only approximately $30 \%$ of patients present with stage I/II disease by PET scan (15). The median age at diagnosis is 66 years, with most patients being diagnosed between ages 65-74 years; this older population was not included in this trial (16). While the findings of this study are certainly applicable to the patient population evaluated, they are not generalizable to patients older than 60 which make for approximately $50 \%$ of all patients with early-stage DLBCL $(8,9)$.

\section{DISCUSSION}

The FLYER trial was built on the observation (from MInT) that a subset of DLBCL patients with age $\leq 60$, zero IPI risk factors, and non-bulky disease have extraordinarily favorable outcomes, with OS close to $95 \%$ when treated with 6 cycles R-CHOP-21. Although a large fraction of these patients had early-stage DLBCL and could have been treated with CMT, they received the extended chemotherapy regimen, without radiation. This observation raised the question: can patients with very favorable DLBCL be treated with only 4 cycles $\mathrm{R}-\mathrm{CHOP}+2$ additional cycles of rituximab and still have the same excellent outcomes with less toxicity? The answer, provided by the FLYER trial, is yes. What the FLYER trial cannot tell us, because it was not designed to, is whether 4 cycles $\mathrm{R}-\mathrm{CHOP}$ are equivalent to 3-4 cycles R-CHOP plus RT, in this population. As discussed above, evidence surrounding the use of RT in patients with early-stage DLBCL is complex and should be parsed carefully, to ensure the right conclusions are not applied to the wrong patients. To avoid this fallacy, reviewing the specifics of each trial is essential. Studies conducted in the pre-rituximab era (S8736 and GELA LNH-93.4) have lost much of their relevance, not only given lack of rituximab, but also because the diagnostic classification of Bcell lymphoma has changed so dramatically that one cannot be sure the same patients are evaluated in more recent studies (in fact, S8736 likely included some T-cell lymphomas). Guessing the impact of COO and molecular features on the outcomes found in these trials is also difficult, because this information was not available when the trials began. Another issue is patient selection based on definition of bulky disease, risk stratification, and age. The trials discussed above are inconsistent regarding definition of "bulk" and eligibility, and conclusions are often drawn from subset analyses of the entire cohort. Finally, emerging data on the value of iPET in adapting treatment selection post-hoc are gradually making a priori therapeutic planning based on risk stratification less relevant. Thus, NCTN S001 and LYSA/GOELAM 02-03 data provide strong support for a RT-free approach to early-stage DLBCL, for iPET-negative patients, including patients older than 60 , and those with tumor bulk $<10 \mathrm{~cm}$. Of course, this raises the question of quality assurance in PET interpretation, which remains inconsistent. This is where the FLYER study is quite helpful, because it did not have a PET-adapted design. In conclusion, most patients with early-stage DLBCL can be treated with abbreviated R-CHOP without radiation. Patients younger than 60 , with tumors $<7.5 \mathrm{~cm}$, and no IPI risk factors, can be treated with 4 cycles R-CHOP without iPET, based on the FLYER trial. Patients older than 60, with masses $<10 \mathrm{~cm}$, and some IPI risk factors, can be treated with 4 cycles R-CHOP if their iPET is negative. Long-term follow up is necessary. The studies of CMT, both before and after rituximab, suggest a pattern of late relapses, which could be due to a distinct biology. Further characterization of the biologic and clinical diversity of early-stage DLBCL is warranted.

\section{AUTHOR CONTRIBUTIONS}

$\mathrm{KS}$ and $\mathrm{AM}$ wrote and edited the manuscript. $\mathrm{AB}$ and $\mathrm{PP}$ reviewed and edited the manuscript. All authors contributed to the article and approved the submitted version.

\section{FUNDING}

AM is supported by the National Institutes of Health institutional training grant T32GM008562. 


\section{REFERENCES}

1. Bonnet C, Fillet G, Mounier N, Ganem G, Molina TJ, Thiéblemont C, et al. Chop Alone Compared With Chop Plus Radiotherapy for Localized Aggressive Lymphoma in Elderly Patients: A Study by the Groupe d'Etude des Lymphomes de l'Adulte. JCO (2007) 25(7):787-92. doi: 10.1200/ JCO.2006.07.0722

2. Habermann TM, Weller EA, Morrison VA, Gascoyne RD, Cassileth PA, Cohn JB, et al. Rituximab-CHOP Versus Chop Alone or With Maintenance Rituximab in Older Patients With Diffuse Large B-Cell Lymphoma. JCO (2006) 24(19):3121-7. doi: 10.1200/JCO.2005.05.1003

3. Pfreundschuh M, Trümper L, Osterborg A, Pettengell R, Trneny M, Imrie K, et al. CHOP-Like Chemotherapy Plus Rituximab Versus CHOP-like Chemotherapy Alone in Young Patients With Good-Prognosis Diffuse large-B-cell Lymphoma: A Randomised Controlled Trial by the MabThera International Trial (MinT) Group. Lancet Oncol (2006) 7(5):379-91. doi: 10.1016/S1470-2045(06)70664-7

4. Poeschel V, Held G, Ziepert M, Witzens-Harig M, Holte H, Thurner L, et al. Four Versus Six Cycles of CHOP Chemotherapy in Combination With Six Applications of Rituximab in Patients With Aggressive B-cell Lymphoma With Favourable Prognosis (FLYER): A Randomised, Phase 3, Non-Inferiority Trial. Lancet (2019) 394:2271-81. doi: 10.1016/S0140-6736(19)33008-9

5. Cheson BD, Horning SJ, Coiffier B, Shipp MA, Fisher RI, Connors JM, et al. Report of an International Workshop to Standardize Response Criteria for non-Hodgkin's Lymphomas. JCO (1999) 17(4):1244. doi: 10.1200/ JCO.1999.17.4.1244

6. Miller TP, Dahlberg S, Cassady JR, Adelstein DJ, Spier CM, Grogan TM, et al. Chemotherapy Alone Compared With Chemotherapy Plus Radiotherapy for Localized Intermediate- and High-Grade Non-Hodgkin's Lymphoma. NEJM (1998) 339):21-6. doi: 10.1056/NEJM199807023390104

7. Persky DO, Unger JM, Spier CM, Stea B, LeBlanc M, McCarty MJ, et al. Phase II Study of Rituximab Plus Three Cycles of CHOP and Involved-Field Radiotherapy for Patients With Limited- Stage Aggressive B-Cell Lymphoma: Southwest Oncology Group Study 0014. JCO (2007) 26 (14):2258-63. doi: 10.1200/JCO.2007.13.6929

8. Lamy T, Damaj G, Soubeyran P, Gyan E, Cartron G, Bouabdallah K, et al. RCHOP 14 With or Without Radiotherapy in Nonbulky Limited-Stage Di Ff Use Large B-cell Lymphoma. Blood (2018) 131(2):174-81. doi: 10.1182/ blood-2017-07-793984

9. Persky DO, Li H, Stephens DM, Park SI, Bartlett NL, Swinnen LJ, et al. Positron Emission Tomography-Directed Therapy for Patients With Limited-Stage Diffuse Large B-Cell Lymphoma: Results of Intergroup National Clinical Trials Network Study S1001. JCO (2020) 38(26):3003-11. doi: 10.1200/JCO.20.00999
10. Barraclough A, Alzahrani M, Ettrup MS, Bishton M, van Vliet C, Farinha P, et al. OO and MYC / BCL2 Status do Not Predict Outcome Among Patients With Stage I / II DLBCL: A Retrospective Multicenter Study. Blood Adv (2019) 3(13):1-3. doi: 10.1182/bloodadvances.2019000251

11. Kumar A, Lunning MA, Zhang Z, Migliacci JC, Moskowitz CH, Zelenetz AD, et al. Excellent Outcomes and Lack of Prognostic Impact of Cell of Origin for Localized Diffuse Large B-Cell Lymphoma in the Rituximab Era. BJH (2015) 171(5):776-83. doi: 10.1111/bjh.13766

12. Torka P, Kothari SK, Sundaram S, Li S, Medeiros LJ, Ayers EC, et al. Outcomes of Patients With Limited-Stage Aggressive Large B-cell Lymphoma With High-Risk Cytogenetics. Blood Adv (2020) 4(2):253-62. doi: 10.1182/bloodadvances.2019000875

13. Youn P, Cummings MA, Dhakal S, Burack WR, Casulo PMB C, Friedberg JW, et al. Prognostic Impact of Cell of Origin in Limited-Stage Diffuse Large B-Cell Lymphoma Treated With R-Chop With or Without Radiation Therapy. Int J Radiat Oncol (2016) 96(2):E495. doi: 10.1016/ j.ijrobp.2016.06.1871

14. Stephens DM, Li H, Leblanc ML, Puvvada SD, Persky D, Friedberg JW, et al. Continued Risk of Relapse Independent of Treatment Modality in LimitedStage Diffuse Large B-Cell Lymphoma: Final and Long-Term Analysis of Southwest Oncology Group Study S8736. JCO (2016) 34(25):2997-3004. doi: 10.1200/JCO.2015.65.4582

15. El-galaly TC, Villa D, Alzahrani M, Hansen JW, Sehn LH, Wilson D, et al. Outcome Prediction by Extranodal Involvement, IPI, R-IPI, and NCCN-IPI in the PET/CT and Rituximab Era: A Danish-Canadian Study of 443 Patients With Diffuse-Large B-cell Lymphoma. AJH (2015) 90(11):1041-6. doi: 10.1002/ajh.24169

16. Teras LR, Desantis CE, Cerhan JR, Morton LM, Jemal A, Flowers CR. 2016 Us Lymphoid Malignancy Statistics by World Health Organization Subtypes. CA Cancer J Clin (2016) 66: (6):443-59. doi: 10.3322/caac.21357

Conflict of Interest: The authors declare that the research was conducted in the absence of any commercial or financial relationships that could be construed as a potential conflict of interest.

The handling editor declared a shared affiliation with the authors at the time of review.

Copyright (c) 2021 Sokol, McBride, Binder and Porcu. This is an open-access article distributed under the terms of the Creative Commons Attribution License (CC BY). The use, distribution or reproduction in other forums is permitted, provided the original author(s) and the copyright owner(s) are credited and that the original publication in this journal is cited, in accordance with accepted academic practice. No use, distribution or reproduction is permitted which does not comply with these terms. 